\title{
Architettura fortificata tra conservazione e riuso: i progetti di restauro novecenteschi del forte di Castelfranco a Finale Ligure
}

Fortified architecture between preservation and reuse strategies: the twentieth century restoration projects of Castelfranco in Finale Ligure

\author{
Enrica Brusa $^{\text {a }}$, Chiara Stanga ${ }^{\text {b }}$ \\ Dipartimento di Architettura e Studi Urbani - Politecnico di Milano, Milan, Italy \\ a enrica.brusa@polimi.it; ${ }^{\mathrm{b}}$ chiara.stanga@polimi.it
}

\begin{abstract}
The town of Finale Ligure, situated on the western coast of Liguria, was the site of the Del Carretto Marquisate until the sixteenth century. After that, it was under the control of the Spanish Crown (seventeenth century) and it has been an independent territory of the Republic of Genoa for a long time. The three castles were built on the top of Finale hills and they were the symbol of its independence. Gavone castle, established on the top of the historical town, has been the site of the Marquisate since the twelfth century. S. Giovanni castle was built by the Spanish in order to improve the town defensive system in the second half of the seventeenth century. Castelfranco, built by the Genoese in the fourteenth century, was rehashed many times by the Spanish and in the nineteenth century by the Savoia family.

The three castles still recall these historical events and are therefore witnesses of the Finale present and past history. They are the result of the different transformations occurred over the centuries. In recent times, Castelfranco has been opened to the public and today it houses art exhibitions and cultural events. The restoration of the castle is the last step of a long-lasting rehabilitation project history that has been developed since the 1900s, when the Municipality suggested to turn it into a hotel. The article analyses the restoration projects of Castelfranco that have been carried out in the first half of the twentieth century, which had different methodologies and approaches. Though this study the article highlights the perception that the town had about the castle, identifying the changes in the balance between reuse and conservation strategies after the first Italian preservation laws.
\end{abstract}

Keywords: Finale Ligure, fortified heritage, Castelfranco, restoration project.

\section{Introduzione}

La Fortezza di Castelfranco è uno dei tre castelli presenti nella città di Finale Ligure (SV). Il forte, costruito nel 1365 dalla Repubblica di Genova come presidio militare contro il dominio dei marchesi Del Carretto e più volte ricostruito, rappresenta l'unico forte della frazione di Finalmarina, ed è situato sull'altura del monte Gottaro, prospiciente il mare. Altri due castelli -

Castel Gavone e Castel S. Giovanni- si trovano invece verso l'interno, a difesa della frazione di Finalborgo. La presenza di un numero così elevato di fortilizi in una cittadina non molto grande come Finale è dovuta alla particolare posizione dell'abitato, dal quale era possibile raggiungere comodamente sia il mare che, via terra, Alessandria e Milano (Fior and Roncai, 2007). 
Così, per la sua importanza strategica, il territorio del Finale fu a lungo conteso tra la signoria locale dei marchesi Del Carretto e la Repubblica di Genova; contesa cui nel XVII secolo si aggiunse poi anche la Spagna.

I tre castelli, costruiti in tempi e per scopi diversi, costituiscono dunque un'importante testimonianza della storia politica del Finale. Tuttavia, ormai privi di un reale scopo difensivo e in alcuni casi irrimediabilmente danneggiati dai combattimenti delle guerre pre-unitarie, essi rimasero a lungo in abbandono, quasi come fossero semplici elementi di secondo piano del paesaggio.

Per quanto riguarda il forte di Castelfranco, questo venne disarmato all'inizio del XIX secolo, per poi essere adibito, verso la metà dello stesso secolo, a reclusorio maschile "per i condannati cronici". La fortezza ricoprì questa funzione fino ai primi anni del '900, quando, su richiesta dell'amministrazione comunale di Finalmarina ${ }^{1}$, iniziò una graduale dismissione (Berruti, 2014, p. 112). Utilizzato ancora come prigione durante la Prima guerra mondiale, fu infine definitivamente abbandonato nel $1920 .^{2}$

Oggi, gli spazi di Castelfranco sono stati quasi interamente recuperati, consentendone l'apertura al pubblico durante l'intero anno grazie ad un progetto di valorizzazione e ad un programma di iniziative di diverso genere, che lo hanno reso un luogo di ritrovo sia per gli abitanti che per i turisti del Finale (Brusa, Stanga, 2019). Tuttavia, il percorso di valorizzazione della fortezza ha avuto inizio parecchi anni prima della riapertura ufficiale, e diversi sono stati i progetti e le proposte che durante il Novecento hanno interessato gli spazi dell'ex-fortezza, spesso senza un esito concreto.

Tra i diversi progetti per il riuso di Castelfranco, l'articolo ha scelto di illustrare le soluzioni che furono progettate tra le due guerre mondiali, con l'obiettivo di metter in luce quale fosse l'idea di tutela prevalente in quegli anni. Per fare questo, sono stati analizzati i progetti presentati all'Ufficio dei Monumenti della Liguria, tuttora conservati nell'archivio della Soprintendenza di Genova. Il periodo temporale analizzato si sviluppa quindi lungo due decenni, compresi tra gli anni '20 e gli anni '30 del secolo scorso. Durante questo periodo furono proposte due diverse soluzioni: la trasformazione dell'ex-forte in un albergo e, poi, in un giardino pubblico.

L'illustrazione dei progetti, presentati con maggiore dettaglio nel quarto capitolo, è preceduta da alcuni paragrafi relativi alle norme di tutela in vigore della prima metà del '900 (capitolo 2) ed all'illustrazione della situazione turistica a Finale durante i primi anni del '900 (capitolo 3).

\section{Leggi di tutela dei monumenti (1909-1939)}

Il primo testo legislativo unitario per la tutela dei monumenti italiani fu il testo n.185, promulgato nel 1902. Questa prima legge, integrata una prima volta dal testo lgs. n.386 del 1907, fu poi nuovamente modificata dalla legge $\mathrm{n}^{\circ} 364$ del 1909 ed, in seguito, ancora dalla breve legge $\mathrm{n}^{\circ} 688$ del 1912, che includeva nella tutela anche le "bellezze naturali", quali parchi e giardini.

Rispetto a quello del 1902, le principali modifiche apportate dal testo di legge del 1909 riguardarono il metodo riconosciuto per l'individuazione dei beni da tutelare -tramite notifica diretta e non più attraverso un catalogo censorio- e le condizioni per l'esportazione dei beni privati (Settis, 2010). La legge stabiliva che dovessero essere sottoposte a tutela "le cose mobili e immobili che avessero interesse storico, archeologico, paletnologico o artistico". Proprio l'uso del termine "cose", invece che quello di "monumenti, immobili e oggetti mobili" usato nella legge 185/1902, faceva dunque riferimento ad una più ampia accezione del termine, derivante dalla consapevolezza dell'importanza di inclusione, tra i beni da tutelare, anche di quelli paesaggistici.

Anche Castelfranco era stato sottoposto a tutela come conseguenza delle leggi del 1902 e del 1909 , le cui notifiche erano state registrate, rispettivamente, il 12/03/1910 ed il 18/12/1920. Inoltre, vale la pena sottolineare come dai documenti ministeriali esaminati traspaia effettivamente una notevole sensibilità verso l'importanza da attribuire alla componente paesaggistica: più volte infatti, come meglio si avrà modo di vedere più avanti, si incontra un richiamo alla "bellezza paesaggistica" del sito, e 
vengono altresì prescritte, per il progetto di ogni nuova costruzione, alcune disposizioni che ne garantiscano l'integrità e l'armoniosità delle forme e delle prospettive con le mura esistenti.

Per quanto invece riguarda la struttura degli uffici di tutela in vigore durante il periodo esaminato, questa si era già sviluppata in maniera sistematica e "completa": le Soprintendenze, istituite ufficialmente con la legge nº38 del 1907 che le poneva alle dipendenze del Ministero della Pubblica Istruzione, distinguendole per ambiti di competenza -S. ai monumenti; S. agli scavi e ai musei archeologici; S. alle gallerie, ai musei, ed agli oggetti d'arte-, garantivano infatti la presenza a carattere "regionale" sul territorio italiano, e potevano ancora contare, a livello locale, anche su alcuni funzionari di supporto: gli "Ispettori agli scavi e ai monumenti" (Dalla Negra, 1992).

Nel caso in esame, dunque, la vigilanza sul rispetto delle norme della tutela era garantita, oltre che dalla presenza di un "Ispettore" nello stesso territorio del Finale, dalla vigilanza esercitata dallo "Ufficio per i monumenti della Liguria" di Genova, una sezione staccata della "Regia Soprintendenza per il Piemonte e la Liguria" di Torino.

\section{Finale agli inizi del ' 900}

Un'altra questione particolarmente rilevante che è emersa dallo studio dei documenti relativi ai progetti di recupero per Castelfranco è quella del fenomeno turistico: quasi tutti gli interventi progettati vennero infatti pensati con una finalità di valorizzazione "turistica" del sito. I nuovi progetti per il riutilizzo della fortezza sarebbero stati, nelle intenzioni dell'amministrazione di Finale Ligure, a beneficio dei forestieri e, in conseguenza, anche della cittadinanza.

Come altre cittadine della Liguria, anche Finale si era infatti aperta al fenomeno del turismo, un'attività che, nelle città vicine al confine con la Francia, si era sviluppata sin dalla seconda metà dell'800 (Scattarreggia, 1986; Boyer, 1997). Il turismo era in realtà arrivato a Finale con qualche anno di ritardo rispetto alle località più occidentali della "Riviera", probabilmente per la mancanza di facili collegamenti con le cit- tà più grandi, e per la vocazione prevalentemente pesco-colturale e di commercio marittimo della città. I primi cambiamenti si avvertirono dopo il 1872 , anno in cui venne inaugurata la linea ferroviaria che ben presto sostituì le infrastrutture tradizionali per il trasporto delle merci, danneggiando così le attività di cabotaggio che costituivano una parte rilevante del reddito delle famiglie locali. La trasformazione degli equilibri economici inizialmente impoverì sensibilmente larga parte della popolazione, e fu proprio a causa di questi cambiamenti che l'amministrazione iniziò a cercare nuove fonti di reddito per la popolazione (Berruti, 2014). I primi dibattiti sull'opportunità di convertire l'economia locale verso quella dell'industria 'del forestiero' apparvero così alla fine dell'Ottocento ed è significativo notare come proprio in quegli anni maturasse una prima proposta di realizzazione di un "albergo grandioso", capace di fornire da solo "larghi vantaggi" per la popolazione (Berruti, 2014, p. 100). Le proposte di quel periodo per la sistemazione della città a favore dei villeggianti non ebbero però il tempo di trovare un effettivo seguito, a causa sia delle scarse risorse finanziarie comunali, sia dell'improvviso sopraggiungere della Guerra, che mise fine alle prime speranze di rinnovamento dell'impianto economico ed urbanistico cittadino. Qualche intervento venne comunque portato a termine: come il miglioramento delle condizioni igieniche della città o la creazione del lungomare tra l'abitato di Finalmarina ed il prospiciente arenile, o ancora la richiesta di dismissione del sanatorio militare, presente all'interno di Castelfranco dalla metà dell'XIX secolo.

\subsection{La situazione turistica negli anni ' 20}

Negli anni'20 del 900, corrispondenti al periodo del primo progetto per il recupero di Castelfranco, il turismo balneare era di nuovo in pieno fermento anche nella città di Finale Ligure. Alcuni dati specifici, relativi allo sviluppo locale della "industria del forestiero", si possono ritrovare a partire dall'ultimo biennio degli anni '20, come conseguenza diretta dell'iscrizione del Comune all'Ente Nazionale delle Imprese Turistiche (E.n.i.t.), che avvenne nel 1929 con il riconoscimento ufficiale da parte del Ministero 
dell'Interno del titolo di "stazione di cura, di soggiorno e di svago" (A.S.C.F.L. - fascicolo "Stazione di soggiorno e cura (miscellanea)", 1929).

Negli archivi comunali si conservano così anche i dati relativi al numero di alberghi e pensioni attivi in quegli anni -rispettivamente 12 e 34 nel 1929-, e delle presenze turistiche annuali che, come riportato nella tabella seguente, raggiunsero circa 14000 unità $^{3}$ :

\begin{tabular}{|c|c|c|c|c|c|}
\hline \multicolumn{3}{|c|}{ anno 1928} & \multicolumn{3}{|c|}{ anno 1929} \\
\hline \multirow{2}{*}{ Nazionalità } & \multicolumn{2}{|c|}{ Stagione } & \multirow{2}{*}{ Nazionalità } & \multicolumn{2}{|c|}{ Stagione } \\
\hline & Estiva & Invernale & & Estiva & Invernale \\
\hline Inglesi & 7 & 5 & Inglesi & 9 & 7 \\
\hline Francesi & 31 & 26 & Francesi & 43 & 31 \\
\hline Belgi & 2 & 3 & Belgi & 6 & 4 \\
\hline Tedeschi & 21 & 19 & Tedeschi & 41 & 23 \\
\hline Austriaci & 6 & 8 & Austriaci & 9 & 11 \\
\hline Ungheresi & 5 & 3 & Ungheresi & 4 & 5 \\
\hline Cecoslovacchi & 9 & 2 & Cecoslovacchi & 3 & 3 \\
\hline Jugoslavi & 3 & 1 & Jugoslavi & 4 & 6 \\
\hline $\begin{array}{l}\text { Olandesi = Danesi } \\
\text { scandinavi }\end{array}$ & 6 & 7 & $\begin{array}{l}\text { Olandesi = Danesi } \\
\text { scandinavi }\end{array}$ & 10 & 11 \\
\hline $\begin{array}{l}\text { Spagnuoli e } \\
\text { Portoghesi }\end{array}$ & 17 & 13 & $\begin{array}{l}\text { Spagnuoli e } \\
\text { Portoghesi }\end{array}$ & 22 & 19 \\
\hline Svizzeri & 58 & 42 & Svizzeri & 65 & 53 \\
\hline $\begin{array}{l}\text { Albanesi = Greci, } \\
\text { Bulgari, Rumeni e } \\
\text { Turchi }\end{array}$ & 5 & 3 & $\begin{array}{l}\text { Albanesi = Greci, } \\
\text { Bulgari, Rumeni e } \\
\text { Turchi }\end{array}$ & 4 & 5 \\
\hline Russi & 11 & 7 & Russi & 10 & 11 \\
\hline Polacchi & 6 & 4 & Polacchi & 8 & 7 \\
\hline Egiziani & 13 & 6 & Egiziani & 7 & 5 \\
\hline Nord Americani & - & 3 & Nord Americani & 9 & 7 \\
\hline $\begin{array}{l}\text { Centro e Sud. } \\
\text { Americani }\end{array}$ & 21 & 9 & $\begin{array}{l}\text { Centro e Sud. } \\
\text { Americani }\end{array}$ & 21 & 17 \\
\hline Totale stranieri & 222 & 160 & Totale stranieri & 275 & 225 \\
\hline Totale Italiani & 12438 & 1368 & Totale Italiani & 12086 & 1629 \\
\hline Totale generale & 12660 & 1528 & Totale generale & 12361 & 1854 \\
\hline
\end{tabular}

\section{I progetti di restauro (1924-1939)}

Come visto, Castelfranco si trovava in un'invidiabile posizione: direttamente affacciato sul mare e situato proprio accanto ad un quartiere di eleganti ville dell'inizio del Novecento, sembrava essere la sede più adatta da sfruttare a beneficio della moderna 'industria dei forestieri'. Tuttavia, seppure dismesso, esso era ancora di proprietà del Demanio dello Stato. Per questi motivi, a partire dai primi anni Venti esso fu al centro di una lunga trattativa con il Ministero della Pubblica Istruzione, al quale spettava la decisione finale riguardo la richiesta di acquisto avanzata dal Comune. La risposta positiva del Ministero era stata vincolata all'approvazione di un dettagliato progetto di recupero. Inoltre, in cambio della vendita e del permesso per la realizzazione del progetto, l'amministrazione avrebbe dovuto comunque garantire la conserva- zione e la manutenzione della parte "monumentale" del sito, coincidente con le mura seicentesche, i bastioni e gli spalti del castello.

Di seguito saranno dunque analizzati i progetti di recupero di Castelfranco presentati dal Comune per ottenerne l'autorizzazione all'acquisto, così come è stato possibile ricostruirli attraverso lo studio delle carte conservate nell' archivio della Soprintendenza di Genova.

\subsection{Il grande albergo}

Il problema della mancanza di un grande albergo, già presentatosi, come visto, nel 1900, fu il primo ad essere proposto per la nuova utilizzazione dell'area dell'ex-fortilizio.

Le trattative per la compravendita furono avviate sin dal 1924. Nel 1926, la stessa Soprintendenza, interrogata dal Ministero riguardo la possibilità di cedere Castelfranco al comune di Finale Ligure, si dichiarò favorevole alla cessione, affermando che: "[...] il forte di Castelfranco, data la sua natura e la sua trasformazione in reclusorio non è di quegli immobili che la nostra amministrazione potrebbe prendere in consegna." (A.S.A.B.A.P.L., cartella "MON 21 - I parte", documento 05/11/1926 della Direzione Regionale per le Antichità e le Belle Arti). Essa pose comunque come condizione per la vendita la visione preliminare del progetto di trasformazione in albergo -al quale pure si dimostrava favorevole- per verificare che la nuova costruzione non danneggiasse le antiche mura del forte. Da parte sua, sin dall'inizio il Comune garantì alla Soprintendenza che "I lavori di trasformazione riguarderebbero l'ex-reclusorio, il quale non è mai stato monumento, ma costituisce una deturpazione del monumento." (A.S.A.B.A.P.L., cartella "MON 21 - I parte", documento 03/01/1927 del commissario prefettizio di Finale Ligure al Soprintendente ai Monumenti della Liguria).

In quegli anni, l'edificio ottocentesco dell'ex reclusorio era costituito da diversi caseggiati, sviluppatisi lungo il profilo dei bastioni ed elevatisi di due o tre piani al di sopra di questi; un articolato corpo di fabbrica a due piani era stato inoltre costruito attorno al torrione centrale del forte. Come oggi, l'ingresso al reclusorio avveniva attraverso il portale seicentesco, situato lungo il 
versante occidentale della fortezza e protetto dalla mezzaluna realizzata dall'ing. Beretta. È comunque molto probabile che, in alcune parti, l'edificio si appoggiasse ad alcuni fabbricati preesistenti, costruiti prima che, agli inizi del XIX secolo, la fortezza perdesse il proprio ruolo di difesa militare.

Per la realizzazione del progetto dell'albergo, che venne presentato il 12.01.1928, furono chiamati due giovani ingegneri di Torino, da poco laureatisi in ingegneria civile: gli ingegneri Carlo Brayda e Luigi Arcozzi-Masino (Politecnico di Torino, 1927).

L'edificio da loro progettato era caratterizzato da una pianta trapezoidale con il lato minore rivolto verso il mare. In alzato, l'edificio prevedeva un piano terra dotato di ampie portefinestre, un piano ammezzato per il personale dell'albergo, e quattro piani per le camere, tutte dotate di balcone. Lo stile delle facciate avrebbe voluto richiamarsi, nelle parole dei progettisti, a quello di "un' architettura moderna con linee che ricordano il barocco genovese".

L'ingresso dell'edificio sarebbe stato posto sul lato nord, in corrispondenza di una corte semicircolare, cui si sarebbe giunti attraverso l'apertura di un passaggio nella muratura dei bastioni. Il nuovo ingresso era stato pensato per dare comodo accesso alle automobili, che non sarebbero invece potute passare attraverso lo stretto passaggio dell'ingresso occidentale; mantenuto come semplice 'ingresso di servizio' per i magazzini e le cucine. La scelta di demolire una parte delle mura a nord era stata conseguenza di due considerazioni: da un lato la trasformazione non sarebbe stata direttamente visibile dalla città, dall'altro, a causa della presenza di grandi finestre aperte probabilmente a metà ' 800 , quella porzione delle mura non era più ritenuta realmente "autentica", e sarebbe dunque potuta essere demolita.

Per quanto riguarda l'impianto planimetrico dell'edificio, il progetto prevedeva che al piano terra vi fossero locali di rappresentanza, una grande aula centrale, vaste sale intercomunicanti ed una sala da pranzo "in diretta comunicazione [...] con il piano inferiore contenente la cucina e gli annessi servizi”. Ai piani superiori avrebbero trovato posto complessivamente 112 camere, per un totale di 200 letti. Nella relazione si specificava che: "La pianta del piano tipo è stata studiata coi criteri più moderni adottati dei migliori alberghi stranieri. Ogni camera ha gabinetto da bagno con W.C. particolare [...]. Le camere ad uno e due letti sono tutte dotate di balcone, cosa molto desiderabile in un albergo di riviera." (A.S.A.B.A.P.L., cartella "MON.21 - I parte", Progetto di trasformazione e adattamento dell'ex-forte di Castelfranco a Finale Ligure in un albergo di lusso,12/01/1928).

I progettisti dichiaravano di essere riusciti a progettare un edificio 'in armonia' con i bastioni, rispetto ai quali sarebbe comunque risultato indipendente. Dello stesso parere non fu però lo "Ufficio per i monumenti della Liguria", che si mostrò subito sorpreso dall'audacia del progetto, sottolineando negativamente come fosse "troppo ingente la mole dell'edificio che colla sua importanza architettonica verrebbe ad essere elemento dominante del paesaggio e danno dei bastioni che se pur conservati e restaurati ne diverrebbero elementi secondari." La Soprintendenza chiese dunque di rivedere il progetto, prescrivendo: "un edificio occupante la medesima area di quello già progettato, ma di altezza metà, senza decorazione esterna e mascherato il più possibile da alberi e verde." (A.S.A.B.A.P.L., cartella "MON.21 - I parte", Lettera del 03/06/1928 al Podestà di Finale).

Il dialogo tra le parti continuò per alcuni anni, in un susseguirsi di richieste e suggerimenti quasi mai accolti dalle controparti, fermamente convinte della bontà delle proprie idee. Il progetto venne infine rivisto nel 1932, modificando la pianta dell'edificio, riducendone l'altezza complessiva a $15,70 \mathrm{~m}$, e semplificando l'impianto decorativo della facciata; la discussione si arenò però sull'avanzata proposta di demolizione del torrione centrale di San Bartolomeo. La torre, comunemente considerata come una delle parti più antiche di Castelfranco, risalente alla prima fase di costruzione genovese del 1365 (Colmuto Zanella, 1972), era infatti considerata in maniera opposta dalla Soprintendenza -per la quale avrebbe dovuto costituire il fulcro della nuova costruzione- e dai progettisti- che la considera- 


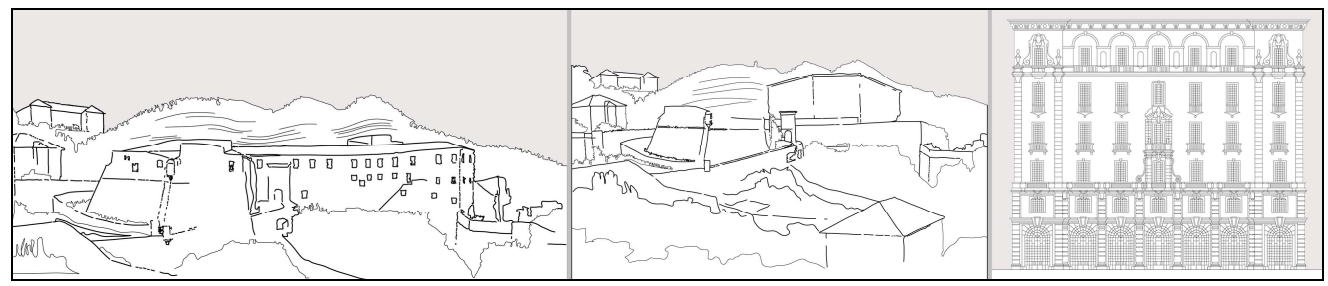

Fig. 1. Ricalco dei disegni del progetto di trasformazione dell'ex-forte di Castelfranco in grande albergo, 1928-1932.

vano invece incompatibile con la modernità caratteristica del grande albergo. Di fronte a questa divergenza di opinioni in merito sia alla conservazione degli elementi caratteristici dell'exforte, sia alla forma del nuovo edificio, vi fu un arresto definitivo delle trattative, senza altre proposte di modifica.

\subsection{Il parco pubblico}

Un secondo progetto di sistemazione venne presentato qualche anno dopo la fine delle trattative per il progetto del grande albergo. L'idea, presentata dall'amministrazione comunale nell'estate del 1937, prevedeva questa volta la creazione di un giardino pubblico all'interno dei bastioni, per la cui realizzazione sarebbe stato sì necessario abbattere le soprastrutture dell'exreclusorio, conservando però interamente il torrione di San Bartolomeo e le mura.

L'area da destinare a giardino pubblico avrebbe avuto una superficie complessiva di $2750 \mathrm{~m}^{2}$, ed all'interno della fortezza, a partire dall'entrata antenuta attraverso il portale esistente sul lato occidentale- sarebbero state create due rampe pedonali, "con selciato di pietrame e cordoni in pietra" che avrebbero garantito l'agevole passeggio tra i dislivelli presenti all'interno. I vialetti pedonali sarebbero stati realizzati invece con del "ghiaietto di mare", e numerosi alberi sarebbero stati piantati anche lungo i controfossi delle mura. Il totale di spesa previsto per la realizzazione del progetto venne quantificato in $170 \mathrm{mi}$ lioni di lire, mentre altri 10 milioni sarebbero dovuti essere impiegati annualmente per la "manutenzione delle opere murarie e dei giardini" (A.S.A.B.A.P.L., cartella "MON.21 - I parte", Verbale di accertamento dello stato di conservazione dell'ex-forte di Castelfranco in Finalmarina, 1936).
Questo secondo progetto venne giudicato positivamente dal Ministero della Pubblica Istruzione, che lo approvò ufficialmente; concedendo così, nel mese di marzo del 1938, la vendita di Castelfranco al comune di Finale.

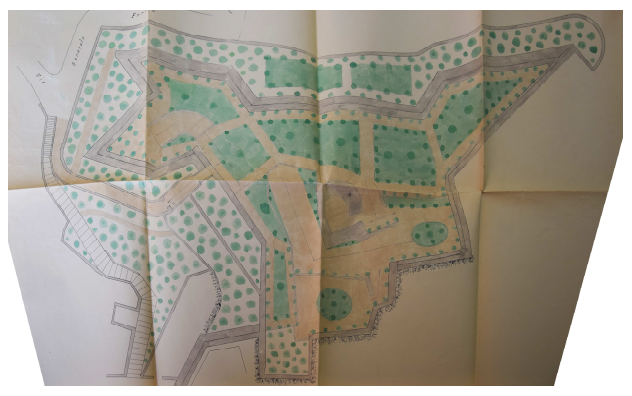

Fig. 2. Planimetria del progetto per la trasformazione di Castelfranco in un giardino pubblico (A.S.A.B.A.P.L., cartella "MON 21 - I parte").

Tuttavia, nonostante le premesse favorevoli, nemmeno in questa occasione il Comune potè procedere alla demolizione dei vecchi edifici. Inizialmente la causa dell'inadempienza fu dovuta alla presenza di alcune famiglie che da qualche anno avevano trovato abusivamente alloggio nei fabbricati abbandonati dell'exreclusorio e per le quali non fu facile trovare una nuova sistemazione. In seguito, lo scoppio della Seconda guerra mondiale paralizzò ulteriormente la realizzazione del progetto, che, come era già successo nel decennio precedente, non ebbe dunque un riscontro concreto.

\section{Quale idea di conservazione?}

Attraverso lo studio dei primi due progetti presentati dal Comune per il recupero del forte dismesso di Castelfranco, è stato possibile ripercorrere l'atteggiamento verso quel particolare tipo di monumento. Ne è emerso così come nel 
secondo quarto del Novecento la chiave di interpretazione dell'edificio consistesse in una sua lettura tipologica, che faceva affermare agli stessi funzionari della Soprintendenza come Castelfranco "d'interessante, dal punto di vista storico militare, non ha che la cinta a speroni sghembi" (A.S.A.B.A.P.L., cartella "MON 21 - I parte", documento 27/12/1932). Il risultato di questo "sguardo" portava così a considerare le superfetazioni di metà Ottocento completamente prive di valore, addirittura degli elementi di "deturpazione" per i bastioni seicenteschi. Questo atteggiamento derivava probabilmente e dall'assenza di avvenimenti storici rilevanti accaduti all'interno delle 'recenti' costruzioni, e dalla vicinanza temporale rispetto alla loro costruzione; fattori che dunque ne limitavano fortemente la percezione di un qualsiasi 'valore di storicità'.

Interessante è poi anche notare come la destinazione d'uso prevista coincidesse in entrambi i casi con un progetto di "valorizzazione turistica": più marcata nel primo caso, di minor impatto nel secondo. Infatti, seppure in entrambi i casi fosse stata approvata la demolizione delle costruzioni al di sopra della linea dei bastioni -ad eccezione della torre di San Bartolomeo- , mentre nel primo progetto si cercò di persuadere $\mathrm{i}$ funzionari del miglioramento che un nuovo edificio avrebbe portato al monumento, nel secondo caso il Comune decise invece di non proporre nessuna rilevante modifica volumetrica dell'area sgomberata dagli edifici ottocenteschi.

Il temporaneo abbandono delle idee di costruzione di un albergo fu dovuta con ogni probabilità alle numerose difficoltà riscontrate durante la presentazione del primo progetto, ma forse anche al progressivo consolidamento della presenza turistica ospitata nelle strutture alberghiere già esistenti, il cui numero era andato via via accrescendosi con il passare degli anni. Il Comune tentò comunque di realizzare un progetto "unico", capace di attirare l'attenzione dei forestieri: se già il viale del lungomare era stato descritto come "senza eguali in tutta la Riviera", e pure se già le altre città balneari si erano dotate di Giardini Botanici, anche Finale Ligure avrebbe avuto il suo parco. E sarebbe stato un lussureggiante giardino all'interno di un ex-fortezza militare.

\section{Conclusioni}

Lo studio qui presentato mostra solo un breve periodo della storia di Castelfranco, di per sé privo di conseguenze concrete per l'edificio e per il paesaggio circostante.

L'effettiva demolizione del reclusorio sarà poi effettivamente compiuta negli anni ' 50 , lasciando però l'ex-fortilizio privo di una reale funzione ancora per lungo tempo. Ciò che qui vale la pena di sottolineare è, da un lato, il grande "motore persuasivo" del turismo che, già pienamente attivo in quegli anni, fu la causa principale dell'ideazione di entrambi i progetti; dall'altro, l'estrema attenzione riscontrata anche in quei lontani anni da parte dei funzionari della Sprintendenza, i quali, pur non riconoscendo alcun valore alle parti costruttive più recenti del forte, controllarono con scrupolosità e competenza che le proposte trasformazioni edilizie non andassero a danneggiarne -secondo i criteri in vigore a quell'epoca- le parti tutelate. Infine, è importante rilevare come il tema della conservazione del paesaggio fosse considerato un tema centrale anche allora; nonostante poi, negli anni successivi, tale consapevolezza non bastò ad impedire che la speculazione edilizia giungesse fino a pochi metri dagli stessi bastioni.

\section{Note}

${ }^{1}$ Le frazioni di Finale (Borgo, Marina e Pia) costituirono tre municipalità autonome sino al 1927.

2 Archivio della Soprintendenza Architettura, Belle Arti e Paesaggio della Liguria [A.S.A.B.A.P.L.], Genova, cartella "MON 21 - I parte", Verbale di accertamento dello stato di conservazione dell'ex-forte di Castelfranco in Finalmarina, 1936.

${ }^{3}$ Archivio Storico del comune di Finale Ligure, Finale Ligure [A.S.C.F.L.], Cartella "Amministrazione", vol. 1-4, fascicolo "Istituti diversi amministrati dal Comune - stazione di soggiorno e cura; anno 1930", Statistica del movimento di forestieri avvenuto nel 1928-1929. 


\section{Ringraziamenti}

Si ringraziano la sig.ra Bianca dell'A.S.C.F.L. ed i responsabili dell'A.S.A.B.A.P.L., sig.ra

Bruno e sig. Coletti, per l'assistenza fornita.

\section{Bibliography}

Berruti, M. (2014). "La scelta. Finale di fronte al futuro", Quaderni dell'Associazione, IV, 4, Associazione Emanuele Celesia Ed., Finale Ligure.

Bono, D. Del. (1987-1988). Progetto di recupero ad uso pubblico e turistico della fortezza di Castelfranco a Finale Ligure, Tesi di laurea, Università degli studi di Genova, Facoltà di Architettura, Genova, pp. 13-15, 24-39, 74121.

Boyer, M. (1997). Il turismo. Dal Grand Tour ai viaggi organizzati, Universale Electa/Gallimard, Trieste, pp. 51-65, 71-87, 94-95

Brusa, E.; Stanga, C. (2019). "Reuse strategies and conservation practices for the fortified architecture. An example from the Liguria region: Finale and its fortresses", in Il patrimonio culturale in mutamento. Le sfide dell'uso. Proceedings of the XXXV International conference 'Scienza e Beni Culturali', 01-05/07/2019, Arcadia Ricerche Ed., Treviso, pp. 773-783.

Colmuto Zanella, G. (1972). "La provincia di Savona", in Bona, E., ed., I castelli della Liguria. Architettura fortificata ligure, Stringa Editore, Genova, vol.1, pp. 220-245, 339-347.

Dalla Negra, R. (1992). "La riforma del servizio di tutela (1902-1915)", in Bencivenni, M.; Dalla Negra, R.; Grifoni, P., eds., Monumenti e istituzioni. Parte seconda: il decollo e la riforma del servizio di Tutela dei monumenti in Italia. 1880-1915, Alinea editrice, Firenze, pp. 183-211.

Fior, M.; Roncai, L. (2007). "Strade, porti, fortificazioni e canali: Finale nel quadro della logistica militare della Lombardia Spagnola", in Peano Cavasola, A., ed., Finale porto di Fiandra, briglia di Genova, Finale Ligure: Centro Storico del Finale, pp. 151-172.

Forni, M. (2017). "Contributi Milanesi alla manualistica sugli alberghi negli ultimi decenni dell'Ottocento", in Belli, G.; Capano, F.; Pascariello, M.I., eds., La città, il viaggio, il turismo; percezione, produzione e trasformazione, Proceedings of the conference, Cirice edizioni, Napoli, pp. 1299-1304.

Pertot, G. (2018). "Archeologia della distruzione: i seicenteschi 'Castelli del Mare' presso Castelfranco, a Finale Ligure (SV). Individuazione dell'impianto e dei resti di una delle più imponenti fortezze del Ponente, contributo per la salvaguardia e la valorizzazione di un sito fragile e dimenticato", in Marotta, A.; Spallone, R., eds., FORTMED 2018. Defensive architecture of the mediterranean, vol. IX, pp. 1317-1324.

Politecnico di Torino (1927). "Allievi che conseguirono la laurea di ingegnere o di architetto negli anni dal 1916 al 1926", in Annuario del Regio Politecnico di Torino, Torino, pp. 348, 351.

Scattarreggia, M. (1986). Sanremo 1815-1915. Turismo e trasformazioni territoriali, Franco Angeli/Storia, Milano, pp. 15-54.

Settis, S. (2010). "Cultura ed etica della tutela: una storia italiana", in Paesaggio, Costituzione, Cemento. La battaglia per l'ambiente contro il degrado civile, Einaudi Ed., Torino, pp. 110-136. 